\title{
Evaluating the Efficiency of different Feature Sets on Brain Tumor Classification in MR Images
}

\author{
Engy N. Eltayeb \\ Department of Biomedical \\ Engineering \\ Faculty of Engineering, \\ Helwan University, Cairo, Egypt
}

\author{
Nancy M. Salem \\ Department of Biomedical \\ Engineering \\ Faculty of Engineering, \\ Helwan University, Cairo, Egypt
}

\author{
Walid Al-Atabany \\ Department of Biomedical \\ Engineering \\ Faculty of Engineering, \\ Helwan University, Cairo, Egypt
}

\begin{abstract}
In this paper, a study for evaluating the efficacy of different feature sets that used brain tumor classification is presented. Different features sets are extracted as shape, $1^{\text {st }}$ order texture features (FOS), $2^{\text {nd }}$ order (GLCM, GLRLM), boundary features, and wavelet-based features. The brain tumors are extracted using the k-means clustering algorithm. Then different classifiers such as Artificial Neural Network (ANN), K-Nearest Neighbor (KNN), and Support Vector Machine (SVM) were used in the classification process.

A set of 65 real and simulated (Flair modality) MRI images from multimodal brain tumor image segmentation benchmark (BRATS) organized by MICCAI 2012 challenge is used for performance evaluation. The overall segmentation results for the 65 volumes are $90.15 \pm 0.12$. For the Feature sets efficacy step, the highest accuracy of $94.74 \%$ is achieved by the SVM when using the wavelet-based features. The lowest accuracy achieved by the three classifiers obtained when using the second order texture features..
\end{abstract}

\section{Keywords}

Brain tumor segmentation, Feature extraction, Wavelet Transform

\section{INTRODUCTION}

Brain tumor detection and classification using image processing techniques is important for the early detection of brain tumor. Magnetic Resonance Imaging (MRI) is a preferred type of imaging modality as it maps the tumor changes and clearly indicates the tumor region $[1,2]$. The process of brain tumor segmentation is a challenging step as it has weak boundaries and inhomogeneous intensities [3].

Automated tumor segmentation methods proposed in the literature can be classified into conventional, classification, clustering and deformable model methods [4]. In conventional methods, image processing methods (such as edge detection and region growing) based on gray intensities of images [5] are used. As a result, these methods were used either as a preprocessing step in the segmentation of brain tumor or refinement step [4]. Recently, these techniques have been combined with artificial neural networks (ANNs) [5], genetic algorithm (GA) [6], fuzzy logic [7], and Markov model.

Supervised techniques such as ANN and support vector machine (SVM) are used in classification methods. Clustering approaches are widely used in tumor segmentation and based on Fuzzy C-means or k-means clustering [8-11]. Coloredbased segmentation methods are also used in the segmentation step [12]. The aim of color translation from gray level MR image into color space image is to obtain more useful feature to achieve good segmentation.

Many feature extraction and classification methods are proposed in the literature for classification and characterization of brain tumors. Zarandi et al. [13] proposed an automated system for diagnosing brain tumors using the framework of fuzzy rules to handle the mass effect and age of brain information as vital features for identifying benign and malignant tumor. But these features are not enough to classify tumors as there are also other important features such as morphological, texture and wavelet features which can utilize more accurate results in classification of tumors.

In other work presented by Arizmendi et al. [14], the Discrete Wavelet Transform (DWT) is used in conjunction with the Bayesian neural network. Authors in [15] developed an algorithm for discriminating between benign or malignant brain tumors on MR image based on texture features. Classification was performed by probabilistic neural network. The achieved accuracy by this system was $94 \%$. However this accuracy could be improved by using several features instead of single feature.

In [16] the authors presented automated CAD system capable of segmentation and classify brain tumor from MRI images with high accuracy. Shape, boundary, wavelet energy, first order statistic (FOS) and second order texture features (GLCM) are used and then ranked. This system based in ensemble classifier used to classify or characterize the tumors as benign or malignant. The features were ranked and then a classifier is used to characterize the tumor.

Feature selection play important role in many classification problems [17-19]. There are several algorithms proposed for feature selection such as absolute value two-sample t-test with pooled variance estimate, principal component analysis (PCA), independent component analysis (ICA) and genetic algorithm.

In this paper, the effect of using different feature sets on the classification performance is presented. The paper is organized as follows: Section 2 presents the different feature sets and the proposed method for tumor segmentation and classification. Results are discussed section 3. Conclusion is given in Section 4

\section{METHODOLOGY}

The aim of this work is to compare different features sets in generating predictions and classifications of brain tumors 


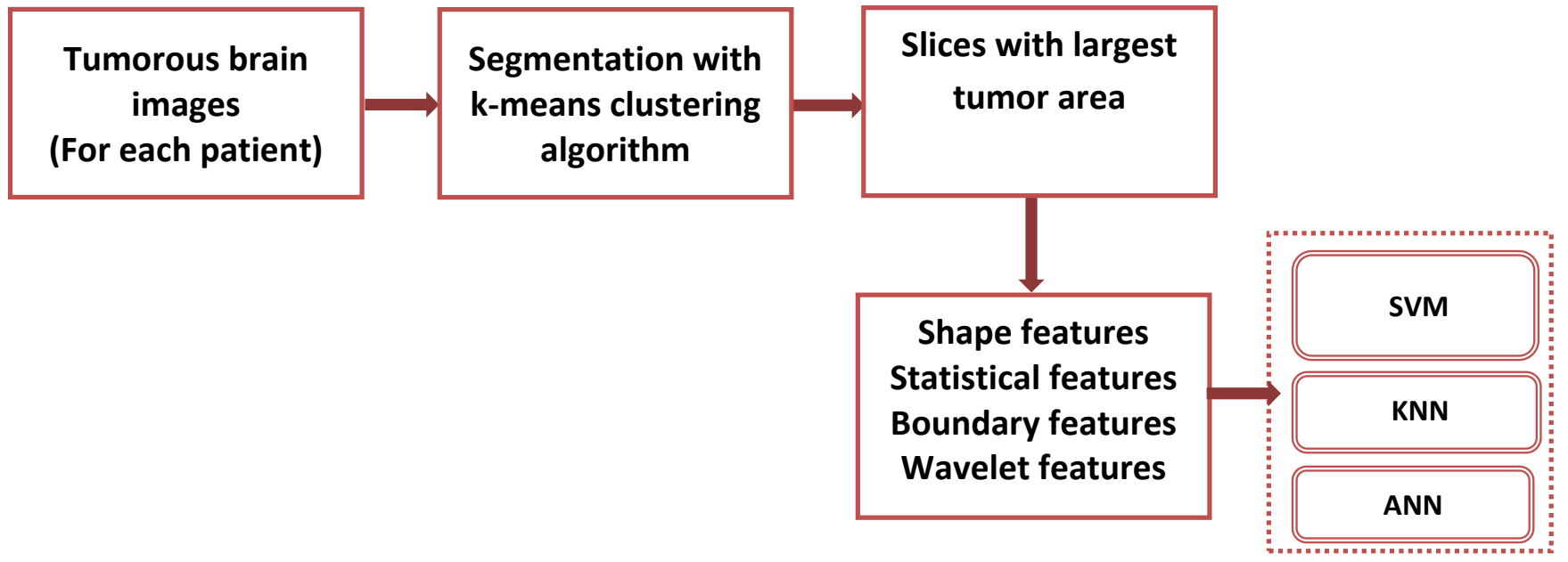

Fig 1: Block diagram of the proposed system.

The proposed system is illustrated in ERROR! REFERENCE SOURCE NOT FOUND..First step is to segment the brain tumor from tumorous slices for each patient, and then slice with largest tumor area is extracted. Finally the efficiency of different feature sets on the classification results is performed.

The segmentation step is a multi-step process, in which the tumorous images are applied to anisotropic diffusion filter (ADF) [8] to smooth intra-region while maintaining objects boundaries intact. Then selecting required features for clustering process by converting the gray-level brain image into CIElab color model ( $\left.\mathrm{L}^{*} \mathrm{a} * \mathrm{~b}\right)$. Finally brain tumor is extracted using k-means with validity index clustering method.

The second step is feature sets efficacy evaluation step .The extracted features are morphological features, statistical features (1st order features, second order (GLCM, GLRLM) features), wavelet features and boundary features. Different feature sets are extracted. The effectiveness of different feature sets is investigated individually. Finally, each feature set vector is applied to three different classifiers such as ANN, SVM, and KNN.

\subsection{SEGMENTATION OF BRAIN TUMOR}

The anisotropic diffusion filter is used to enhance the tumorous image and preserve borders of tumor. This filter is applied according to the following equation 1:

$$
\frac{\delta}{\delta t} I(\overline{\mathrm{A}}, t)=\nabla \cdot(c(\overline{\mathrm{A}}, t) \nabla I(\overline{\mathrm{A}}, t))
$$

Where $I(\overline{\mathrm{A}}, t)$ is the input image, $\overline{\mathrm{A}}$ is the axis of the image, $\mathrm{t}$ is iteration step and $c(\overline{\mathrm{A}}, t)$ is the diffusion coefficient.

$$
c(\overline{\mathrm{A}}, t)=\exp \left(\mathrm{l}-\left(\frac{|\nabla I(\overline{\mathrm{A}}, t)|}{\sqrt{2 K}}\right)^{2}\right)
$$

$\mathrm{K}$ is the diffusion constant and it controls the behavior of the filter.

A color transformation function is applied to utilize more information from tumorous images instead in the gray level.

First, the gray level image is converted into RGB image in order to utilize useful features and enhance the visual density [20] and then converted to a CIELab color model (L*a*b*) [21].
Finally, the K-means algorithm is used to find the cluster that contains the tumor in the brain image. In this clustering step, the $\mathrm{K}$-means is used.

\subsection{FEATURE SETS EFFICACY EVALUATION}

In this paper, we compare four sets of features which are shape features, statistical (texture) features $\left(1^{\text {st }}\right.$ order, $2^{\text {nd }}$ order (GLCM,GLRLM) features, Wavelet energy and boundary feature using non-linear technique Haussdorf fractal dimension. The output of segmentation is a set of segmented tumorous slices, so the slice containing the largest area of the tumor is detected/selected from that set of slices for each patient. The chosen (detected) slice contains the maximum possible information of tumor.

Each feature set is ranked individually using absolute value two-sample t-test with pooled variance estimate. It is a parametric test that compares the location parameter of two independent data patterns [19].

\subsubsection{Feature Extraction}

\subsubsection{Morphological or shape features}

The changes in the anatomical shapes of the brain tissue observed on MRI images such as lesions, edema, necrosis inside lesion [17] gives necessary information about the growth of tumors in brain tissues. This information aid to identify the type and grade of brain tumor as in meningioma tumors (low grade) the shape is regular with sharp boundary in spite of convoluted shape and diffuse boundary in glioblastoma multiform (GBM) which considered as high grade tumor.

In this paper, we extracted 15 shape features based on geometric parameters such as perimeter, area, major axis, minor axis, thinness ratio, eccentricity, Equidiameter, dispersion, compactness, circularity, roundness, elongation, and shape index are measured to identify the shape of the tumor $[17,18]$.

\subsubsection{Texture (statistical) features}

Texture information can be used to discriminate the tissues of the organ. Here texture features are extracted using 1st order and 2 nd order statistics methods of the tumor region tissue [15-17]. 
First order statistic features (FOS):

In our experiment, we calculated five FOS features which are the average gray level, standard deviation, entropy, skewness, and kurtosis [22].

\section{Second order statistic features:}

The texture characteristics of extracted tumor which correspond to second-order statistics features such as the gray level co-occurrence matrix and gray level run length matrix $[16,19]$. The gray level co-occurrence matrix (GLCM) is a statistical method used to analyze the texture of the image. In our case, 20 features are computed using GLCM matrices. Such as contrast, correlation, energy, homogeneity, cluster shade, dissimilarity, sum average, sum variance and maximal correlation coefficient [23].

The gray level run length GLRLM is a spatial domain statistical method that provides information about the linked length of an individual pixel in a specific direction. The GLRLM is calculated for $0^{\circ}, 45^{\circ}, 90^{\circ}$ and $135^{\circ}$. We extracted seven features using GLRLM matrices a from the gray scale image such as Short Run Emphasis (SRE), Long Run Emphasis (LRE), Gray Level Non-uniformity (GLN), Run Percentage (RP), Run Length Non-uniformity (RLN), Low Gray Level Run Emphasis (LGLRE) and High Gray Level Run Emphasis (HGLRE) [24].

\subsubsection{Wavelet- based features}

The wavelet energy is called wavelet-based feature which reflective the energy distribution in different directions at different resolutions of the image. The wavelet energy was computed for the details sub-band and the approximate subband [16].

\subsubsection{Boundary features}

Characteristics of the brain tumor boundary play an important role in differentiate benign and malignant tumor as a typical benign tumor has a uniform round and smooth boundary. On the other hand, malignant tumor has a speculated and rough boundary [16]. The degree of border irregularity is calculated using modified box-counting method for measuring surface fractal dimension [25].

\subsubsection{Feature Ranking using T-test}

The Hold-Out cross validation method is used. It has the advantage of partition the entire dataset in training and testing as you need. Here we used $70 \%$ of entire data set as training and test with the rest in the phase of classifiers,

in this work, each feature set is ranked individually using absolute value two-sample t-test with pooled variance estimate. It is a simple method for finding important features. It is a parametric test that compares the location parameter of two independent data patterns. The algorithm statistics is given by:

$$
\frac{\bar{x} \cdot \bar{y}}{\sqrt{\frac{s_{x}^{2}}{n}+\frac{s_{y}^{2}}{m}}}
$$

\subsubsection{Classification}

In this step, three different classifiers were tested which are Artificial Neural Network (ANN), K-Nearest Neighbor (KNN), and Support Vector Machine (SVM). ANN is unsupervised learning technique and it is a three-layer feed forward neural network [26] is implemented with $n$ input, $h$ hidden, and one output neuron
K-Nearest neighbor is based on the concept that the instances of data of the same class should be closer in the feature space. As a result, given an input feature vector $\mathrm{X}$ of unknown class, it determines the $\mathrm{k}$ closest training vectors according to similarity measure distance metric (Euclidean), Vector $\mathrm{X}$ is assigned to the class to which the majority of k-nearest neighbors belong [27]

Support vector machine is a supervised learning machine for binary classification problems. Given a training dataset

$\{\mathrm{xi}$, yi $\} \mathrm{Ni}=1$, where $\mathrm{xi} \in \mathrm{Rd}$ is a feature vector and $\mathrm{d}$ the dimension of the input feature vector and yi $\in\{-1,+1\}$ is a class label, SVM identifies the data points near the optimal separating hyperplane, which is called support vectors.

\section{RESULTS AND DISCUSSION}

The proposed system is tested and evaluated using a publicly available dataset; MICCAI 2012 Challenge on Multimodal Brain Tumor Segmentation (Brats) [28]. It consists of 29 realpatients containing 20 high-grade volumes and 9 low-grade volumes. Also simulated Brats dataset consists of 25 highgrade volumes and 25 low-grade volumes. Each volume contains 160 slices of FLAIR MRI images.

The performance of different sets is evaluated using a set of 65 volumes of the Brats dataset (24 real-patients and 41 simulated data.

The main purpose of this paper is to study the efficiency and efficacy of different feature sets in quantification and classification of brain tumors. In this step, we evaluated the ability and proficiency of different feature sets in which those feature categories are widely used in brain tumor classification. Tables 1 and 2 summarize the classification results for each feature set obtained from Flair modality of sixty five real and simulated Brats data sets when using three different classifiers.

It can be observed that the Wavelet-based features (two wavelet energy features) achieve the best performance results using the three classifiers (SVM, ANN, KNN) and also for the sensitivity and specificity of each classifiers. Also it can be seen that in most feature sets, SVM gives highest performance in spite of in some cases SVM classifier sensitivity is very low as in 2nd order statistic features (GLCM, GLRLM) individually.

When comparing the efficiency of the four feature sets, the best results are achieved for the wavelet features by the three classifiers: SVM, ANN, and KNN which is $94.74 \%$ for the SVM classifier. The second best results for 1st order features ( 5 features) achieved by the three classifiers and the results are the same which are $89.47 \%$

In the boundary features using Hausdorff fractal dimensions method the three classifiers give the same accuracy results $84.21 \%$ while the ANN has the lowest sensitivity compared with the other classifiers. 
Table 1: Results of classification using ANN with different feature sets.

\begin{tabular}{|l|l|l|l|}
\hline \multirow{2}{*}{ Features sets } & $\begin{array}{l}\text { Accuracy } \\
\%\end{array}$ & $\begin{array}{l}\text { Sensitivity } \\
\mathbf{\%}\end{array}$ & $\begin{array}{l}\text { Specificity } \\
\mathbf{\%}\end{array}$ \\
\cline { 2 - 4 } & ANN & ANN & ANN \\
\hline FOS & 89.47 & 77.78 & 100 \\
\hline GLCRM & 84.21 & 77.77 & 90 \\
\hline GLRLM order & 78.95 & 77.78 & 80 \\
\hline $\begin{array}{l}\text { 2nd GLCM+GLRL } \\
\text { Over all texture } \\
\text { feature }\end{array}$ & 78.94 & 88.88 & 70 \\
\hline $\begin{array}{l}\text { Wavelet } \\
\text { features }\end{array}$ & $\mathbf{9 4 . 4 7}$ & $\mathbf{1 0 0}$ & $\mathbf{9 0}$ \\
\hline $\begin{array}{l}\text { Boundary } \\
\text { features }\end{array}$ & 84.21 & 77.77 & 90 \\
\hline Shape features & 89.47 & 88.88 & 90 \\
\hline
\end{tabular}

*The values in bold denote cases in which the given feature set yields significantly better results than the other sets.

Table 2: Results of classification using SVM and KNN with different feature sets.

\begin{tabular}{|l|l|l|l|l|l|l|}
\hline \multirow{2}{*}{$\begin{array}{l}\text { Features } \\
\text { sets }\end{array}$} & \multicolumn{2}{|l|}{ Accuracy \% } & \multicolumn{2}{l|}{ Sensitivity \% } & \multicolumn{2}{l|}{ Specificity \% } \\
\cline { 2 - 7 } & SVM & $\begin{array}{l}\text { KN } \\
\text { N }\end{array}$ & SVM & KNN & SVM & KNN \\
\hline FOS & 89.47 & $\begin{array}{l}89.4 \\
7\end{array}$ & 88.89 & 77.78 & 90 & 100 \\
\hline GLCRM & 78.95 & $\begin{array}{l}78.9 \\
5\end{array}$ & 55.56 & 66.67 & 100 & 90 \\
\hline $\begin{array}{l}\text { GLRLM } \\
\text { (GLCM+G } \\
\text { LRLM) }\end{array}$ & 84.21 & $\begin{array}{l}84.2 \\
1\end{array}$ & 77.78 & 77.78 & 90 & 90 \\
\hline $\begin{array}{l}\text { All texture } \\
\text { feature }\end{array}$ & 84.21 & $\begin{array}{l}73.6 \\
8\end{array}$ & 77.78 & 77.78 & 90 & 70 \\
\hline $\begin{array}{l}\text { Wavelet } \\
\text { features }\end{array}$ & $\mathbf{9 4 . 7 4}$ & $\begin{array}{l}89.4 \\
7\end{array}$ & $\mathbf{1 0 0}$ & 88.89 & 90 & $\mathbf{9 0}$ \\
\hline $\begin{array}{l}\text { Boundary } \\
\text { features }\end{array}$ & 84.21 & $\begin{array}{l}84.2 \\
1\end{array}$ & 88.89 & 88.89 & 80 & 80 \\
\hline $\begin{array}{l}\text { Shape } \\
\text { features }\end{array}$ & 84.21 & $\begin{array}{l}89.4 \\
7\end{array}$ & 88.89 & 88.88 & 80 & 90 \\
\hline
\end{tabular}

*The values in bold denote cases in which the given feature set yields significantly better results than the other sets.

From Tables 1 and 2, it can be observed that the lowest results achieved by the SVM and KNN classifiers when using the GLCRM (20 features). The ANN gives its lowest accuracy in 2nd order statistical features (GLCRM+GLRLM) (27 features) and all texture features.
Figures 2, 3, and 4 illustrate the performance of the three classifiers and their sensitivity and specificity achieved using each feature sets.

Figure 5 shows the performance results of the three classifiers (SVM, KNN, ANN) with combination all feature categories and with different number of features ranked by the T-test. The $\mathrm{x}$-axis shows the ranked number of features used for classification, and the y-axis represents the classification accuracy.

It can be seen that, with 30 features of the T-test output, the accuracy of the SVM classifier gives the highest performance $(89.47 \%)$ compared with other two classifiers.

The classification accuracies of ANN is the most stable and gives high performance with almost number of features of Ttest as it gives high performance with $5,10,15,45$ features of T-test which is $89.47 \%$ and lowest performance with 25 features of T-test (78.95\%). While the performance of KNN, with only few features of T-test ( 1 to 15 features) give reasonable accuracy $(84.21 \%)$ then it performance decrease with increasing number of features used.

Table 3, 4, 5 summarize the results of classification using the feature combinations, hold-out cross validation was repeated randomly 25 times using SVM, KNN and ANN classifiers on the 65 cases of Brats dataset.

We can see that the best brain tumor classification accuracy rate achieved by SVM classifier was $89.47 \%$ with two features, GLCRM,GLRLM (2nd order statistical features) while the best performance occurred by ANN classifier was $89.47 \%$ with two different combinations of features which are (DWT, FOS) and (shape, boundary). Finally the best performance of KNN classifier was $89.47 \%$ with DWT, FOS features.

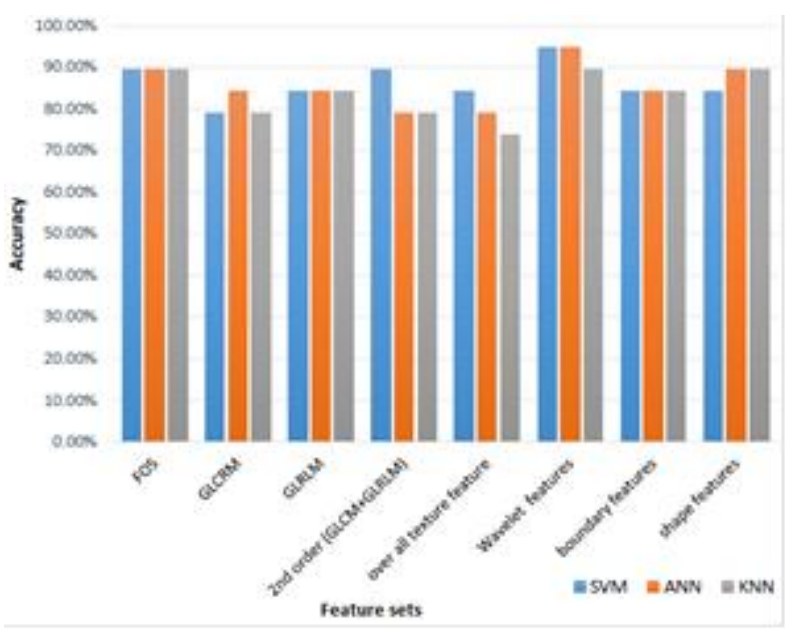

Fig 2: Comparison of accuracy results of three classifiers: SVM, ANN, KNN using different sets of features Obtained from Flair modality of real and simulated data (65 cases). 


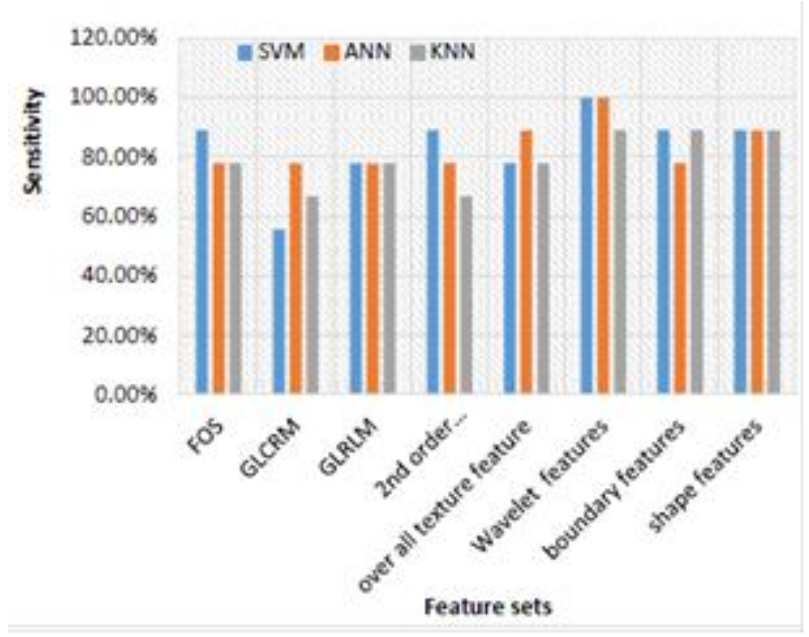

Fig 3: Sensitivity results of three classifiers using different sets of feature obtained from Flair modality of real and simulated data ( 65 cases).

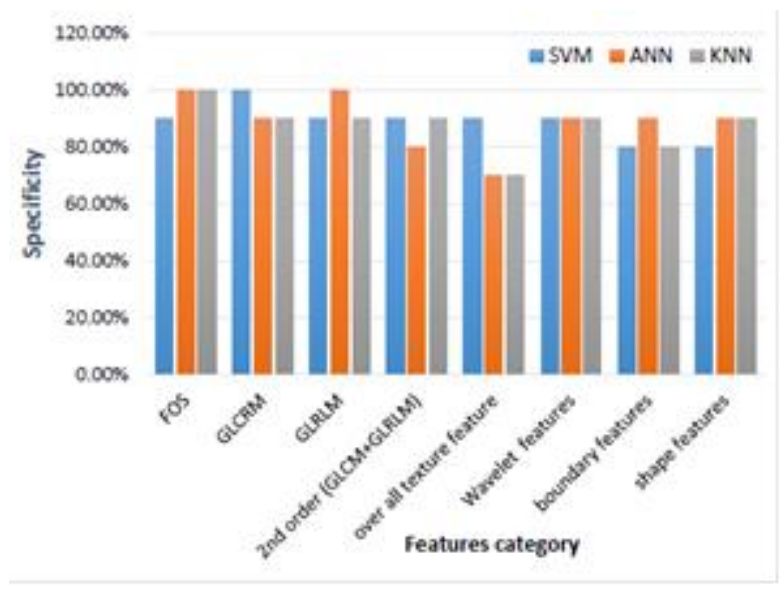

Fig 4: Specificity results of three classifiers using different sets of feature obtained from Flair modality of real and simulated data ( 65 cases).

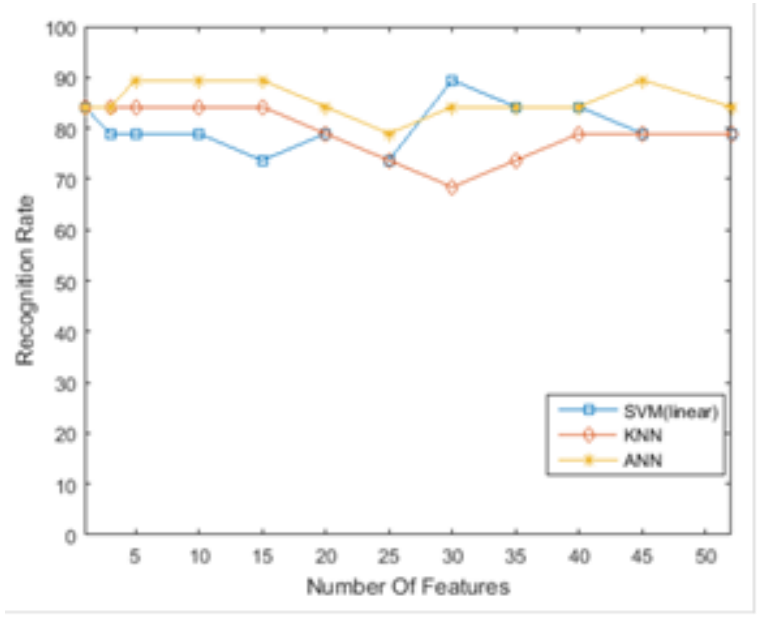

Fig 5: Classification accuracy of SVM, KNN and ANN for different numbers of features selected using T-Test.
Table 3: Best performances of features combinations using SVM (linear) classifier

\begin{tabular}{|c|c|c|c|c|}
\hline \multirow[b]{2}{*}{$\begin{array}{l}\text { No. of } \\
\text { Features }\end{array}$} & \multirow[b]{2}{*}{ Feature combinations } & \multicolumn{3}{|l|}{ SVM } \\
\hline & & $\begin{array}{l}\text { Acc } \\
\%\end{array}$ & $\begin{array}{l}\text { Sen } \\
\%\end{array}$ & $\begin{array}{l}\text { Sp } \\
\%\end{array}$ \\
\hline 17 & DWT, shape & $8^{78.9}$ & 77.78 & 80 \\
\hline 7 & DWT, FOS & $5^{78.9}$ & 88.89 & 70 \\
\hline 20 & shape, FOS & $8^{73.6}$ & 77.78 & 70 \\
\hline 22 & DWT, fOS, shape & $5^{78.9}$ & 88.89 & 70 \\
\hline 3 & boundary, DWT & $1^{84.2}$ & 88.89 & 80 \\
\hline 16 & boundary, shape & $5^{78.9}$ & 77.78 & 80 \\
\hline 6 & boundary, FOS & $5^{78.9}$ & 77.78 & 80 \\
\hline 21 & boundary, FOS, shape & $5^{78.9}$ & 77.78 & 80 \\
\hline 9 & GLRLM,DWT & $5^{78.9}$ & 77.78 & 80 \\
\hline 18 & boundary, DWT, shape & $5^{78.9}$ & 66.89 & 70 \\
\hline 23 & boundary,DWT,shape,FOS & $8^{73.6}$ & 66.67 & 80 \\
\hline 27 & $\begin{array}{l}\text { GLCRM,GLRLM }\left(2^{\text {nd }} \text { order }\right. \\
\text { statistical features })\end{array}$ & $7^{89.4}$ & 88.89 & 90 \\
\hline 32 & All statistical features & $\begin{array}{l}{ }^{84.2} \\
1\end{array}$ & 77.78 & 90 \\
\hline 50 & All features sets $(50)$ & $8^{78.9}$ & 88.89 & 70 \\
\hline
\end{tabular}

Table 4 : Best performance of features combinations using ANN classifier

\begin{tabular}{|l|l|l|l|l|}
\hline \multirow{2}{*}{$\begin{array}{l}\text { No. of } \\
\text { features }\end{array}$} & \multirow{2}{*}{ Feature combinations } & $\begin{array}{l}\text { ANN } \\
\text { Acc }\end{array}$ & $\begin{array}{l}\text { Sen } \\
\mathbf{\%}\end{array}$ & $\begin{array}{l}\text { Sp } \\
\mathbf{\%}\end{array}$ \\
\hline 17 & & 84.21 & 88.88 & 80 \\
\hline 7 & DWT, shape & $\mathbf{8 9 . 4 7}$ & $\mathbf{8 8 . 8 9}$ & $\mathbf{9 0}$ \\
\hline 20 & DWT, FOS & 84.21 & 77.77 & 90 \\
\hline 22 & shape, FOS & 84.21 & 88.88 & 80 \\
\hline 3 & DWT, FOS, shape & 84.21 & 77.77 & 90 \\
\hline 16 & boundary, DWT & $\mathbf{8 9 . 4 7}$ & $\mathbf{8 8 . 8 8}$ & $\mathbf{9 0}$ \\
\hline 6 & boundary, FOS & 78.94 & 88.88 & 70 \\
\hline 21 & boundary, FOS, shape & 84.21 & 77.77 & 90 \\
\hline 9 & GLRLM, DWT & 84.21 & 77.77 & 90 \\
\hline
\end{tabular}




\begin{tabular}{|c|c|c|c|c|}
\hline 18 & boundary, DWT, shape & 84.21 & 88.88 & 80 \\
\hline 23 & boundary,DWT,shape,FOS & 73.68 & 88.88 & 60 \\
\hline 27 & $\begin{array}{l}\text { GLCRM,GLRLM }\left(2^{\text {nd }}\right. \\
\text { order statistical features })\end{array}$ & 78.95 & 77.78 & 80 \\
\hline 32 & All statistical features & 78.94 & 88.88 & 70 \\
\hline 50 & $\begin{array}{l}\text { All features sets } \quad(50 \\
\text { features) }\end{array}$ & 84.21 & 88.88 & 80 \\
\hline
\end{tabular}

Table 5: Best performances of features combinations using KNN classifier

\begin{tabular}{|c|c|c|c|c|}
\hline \multirow[b]{2}{*}{$\begin{array}{l}\text { No. of } \\
\text { features }\end{array}$} & \multirow[b]{2}{*}{ Feature combinations } & \multicolumn{3}{|l|}{ KNN } \\
\hline & & $\begin{array}{l}\text { Acc } \\
\%\end{array}$ & $\begin{array}{l}\text { Sen } \\
\%\end{array}$ & $\begin{array}{l}\text { Sp } \\
\%\end{array}$ \\
\hline 17 & DWT, shape & 78.95 & 77.78 & 80 \\
\hline 7 & DWT, FOS & 89.47 & 88.88 & 90 \\
\hline 20 & shape, FOS & 78.68 & 77.78 & 70 \\
\hline 22 & DWT, FOS, shape & 78.98 & 77.78 & 80 \\
\hline 3 & boundary, DWT & 84.21 & 77.78 & 90 \\
\hline 16 & boundary, shape & 84.21 & 77.78 & 90 \\
\hline 6 & boundary, FOS & 73.68 & 77.78 & 70 \\
\hline 21 & boundary, FOS, shape & 73.68 & 77.78 & 70 \\
\hline 9 & GLRLM,DWT & 78.95 & 66.67 & 90 \\
\hline 18 & boundary, DWT, shape & 84.21 & 77.78 & 90 \\
\hline 23 & boundary,DWT,shape,FOS & 73.68 & 77.78 & 70 \\
\hline 27 & $\begin{array}{l}\text { GLCRM,GLRLM }\left(2^{\text {nd }}\right. \\
\text { order statistical features })\end{array}$ & 78.95 & 66.67 & 90 \\
\hline 32 & All statistical features & 73.68 & 77.78 & 70 \\
\hline 50 & $\begin{array}{l}\text { All features sets } \quad(50 \\
\text { features) }\end{array}$ & 78.95 & 77.78 & 80 \\
\hline
\end{tabular}

\section{CONCLUSION}

In this paper, the evaluation of the proficiency and ability of different and widely used features in classification of brain tumors has been proposed. Features are shape, statistical (FOS, GLCRM, GLRLM), Wavelet-based and boundary using modified counting box FD features. The best performance $94.74 \%$ was achieved by the SVM for the wavelet -based features. The lowest accuracy achieved by the three classifiers obtained when using the second order (GLCRM+ GLRLM) and all texture features which are $78.95 \%$ in GLCRM, 78.95\% in 2nd order statistical (texture) features (GLCRM+GLRLM) and in all texture features and $73.68 \%$ respectively. The final step in this study, we evaluate the best performance for SVM, ANN, KNN classifiers was $89.47 \%$ with different feature categories combinations. In which the best SVM performance classifier with $2^{\text {nd }}$ order texture features (GLCRM, GLRLM) while the ANN gives best performance with (FOS, DWT) and (Boundary, Shape). The KNN gives best performance with (FOS, DWT) combination.

\section{REFERENCES}

[1] A. Drevelegas and N. Papanikolaou, "Imaging modalities in brain tumors," in Imaging of Brain Tumors with Histological Correlations: Springer, pp. 13-33, (2002).

[2] B. H. Menze, A. Jakab, S. Bauer, J. Kalpathy-Cramer, K. Farahani, J. Kirby, Y. Burren, N. Porz, J. Slotboom, and R. Wiest, "The multimodal brain tumor image segmentation benchmark (BRATS)," IEEE Transactions on Medical Imaging, vol. 34, pp. 1993-2024,(2015).

[3] K. Usman and K. Rajpoot, "Brain tumor classification from multi-modality MRI using wavelets and machine learning," Pattern Analysis and Applications, pp. 1-11, (2014).

[4] J. Liu, M. Li, J. Wang, F. Wu, T. Liu, and Y. Pan, "A survey of MRI-based brain tumor segmentation methods," Tsinghua Science and Technology, vol. 19, pp. 578-595, (2014).

[5] H. Tang, E. Wu, Q. Ma, D. Gallagher, G. Perera, and T. Zhuang, "MRI brain image segmentation by multiresolution edge detection and region selection," Computerized Medical Imaging and Graphics, vol. 24, pp. 349-357, (2000).

[6] M. Jafari and S. Kasaei, "Automatic brain tissue detection in MRI images using seeded region growing segmentation and neural network classification," Australian Journal of Basic and Applied Sciences, vol. 5, pp. 1066-1079, (2011)

[7] E.-S. A. El-Dahshan, H. M. Mohsen, K. Revett, and A.B. M. Salem, "Computer-aided diagnosis of human brain tumor through MRI: A survey and a new algorithm," Expert systems with Applications, vol. 41, pp. 55265545, (2014)

[8] Q. Ain, M. A. Jaffar, and T.-S. Choi, "Fuzzy anisotropic diffusion based segmentation and texture based ensemble classification of brain tumor," Applied Soft Computing, vol. 21, pp. 330-340.

[9] S. Bandyopadhyay, "MRI brain image segmentation by fuzzy symmetry based genetic clustering technique," Evol. Comput, pp. 4417-4424, (2007)

[10] J. Selvakumar, A. Lakshmi, and T. Arivoli, "Brain tumor segmentation and its area calculation in brain MR images using K-mean clustering and Fuzzy C-mean algorithm," in Advances in Engineering, Science and Management (ICAESM), 2012 International Conference on, pp. 186190, (2012).

[11] M.-N. Wu, C.-C. Lin, and C.-C. Chang, "Brain tumor detection using color-based k-means clustering segmentation," in Third International Conference on Intelligent Information Hiding and Multimedia Signal Processing, 2007, pp. 245-250, (2007).

[12] M. M. Ahmed and D. B. Mohamad, "Segmentation of brain MR images for tumor extraction by combining kmeans clustering and perona-malik anisotropic diffusion model," International Journal of Image Processing, vol. 2, pp. 27-34, (2008).

[13] F. Zarandi, M.H., Zarinbal, M., Izadi, M.: Systematic image processing for diagnosing brain tumors: a type-II fuzzy expert system approach. Appl. Soft Comput. 11(1), 285-294, (2011). 
[14] C. Arizmendi, A. Vellido, E. Romero, "Binary classification of brain tumors using discrete wavelet transform and energy criteria. In: Proceedings of IEEE Second Latin American Symposium on Circuits and Systems, pp. 1-4, (2011).

[15] Georgiadis, P., Cavouras, D., Kalatzis, I., Daskalakis, A., Kagadis, G.C., Sifaki, K., Malamas, M., Nikiforidis, G., Solomou, E.:Improving brain tumor characterization on MRI by probabilistic neural network and non-linear transformation of textural features. Comput. Methods Programs Biomed. 89(1), 24-32, (2008).

[16] Reddy,Megha P.Arakeriand G.Ram Mohana, Computeraided diagnosis system for tissue characterization. Signal, Image and Video processing vol 9, pp.409-425, (2015)

[17] Verme N., Cowperthwaite M.C., Burnett M.G., Markey M.K. (2014) Image Analysis Techniques for the Quantification of Brain Tumors on MR Images. In: Suzuki K. (eds) Computational Intelligence in Biomedical Imaging. Springer, New York, NY

[18] Kassimi, M.A., El beqqali, O., "3D model retrieval based on semantic and shape indexes". Int. J. Comput. Sci. Issues 8(1), 172-181, (2011).

[19] N. Nabizadeh and M. Kubat, "Brain tumors detection and segmentation in MR images: Gabor wavelet vs. statistical features," Computers \& Electrical Engineering, vol. 45, pp. 286-301, (2015).

[20] M. B. A. Haghighat, A. Aghagolzadeh, and H. Seyedarabi, "A non-reference image fusion metric based on mutual information of image features," Computers \& Electrical Engineering, vol. 37, pp. 744-756, (2011).

[21] C. Connolly and T. Fleiss, "A study of efficiency and accuracy in the transformation from RGB to CIELAB color space," IEEE Transactions on Image Processing, vol. 6, pp. 1046-1048, (1997).

[22] Akilandeswari, U., Nithya, R., Santhi, B.:Reviewon feature extraction methods in pattern classification. Euro. J. Sci. Res. 71(2), 265-272, (2012).

[23] Haralick, R.M., Shanmugam, K., Dinstein, I.: Texture features forimage classification. IEEE Trans. Syst. Man Cybern. 3(6), 610-621, (1973).

[24] Conners RW, Harlow CA. A theoretical comparison of texture algorithm. IEEE Trans Pattern Anal Mach Intell 1980;2:204-22, (1980).

[25] Iftekharuddin, K.M., Jia, W., Marsh, R.: Fractal analysis of tumor in brain MR images. Mach. Vis. Appl. 13, 352$362,(2003)$

[26] Haykin, S.O.: Neural networks and Learning Machines, 3rd edn,Prentice Hall (2008)

[27] Song, Y., Huang, J., Zhou, D., Zha, H., Giles, C.L. IKNN: informative k-nearest neighbor pattern classification, LNCS, Vol. 4702, Springer, pp. 248-264, (2007).

[28] http://www2.imm.dtu.dk/projects/BRATS2012/ 